\title{
Assessment of the Clinical Effects of Aquatic-Based Exercises in the Treatment of Children With Juvenile Dermatomyositis: A $2 \times 2$ Controlled-Crossover Trial
}

\author{
Ahmed SAMHAN ${ }^{1,4}(\mathbb{D})$, Nermeen MOHAMED $^{1,4}(\mathbb{D})$, Ragab ELNAGGAR ${ }^{2,4}\left(\mathbb{D}\right.$, Waleed MAHMOUD ${ }^{3,4}(\mathbb{D}$ \\ ${ }^{1}$ Department of Physical Therapy, New Kasr El-Aini Teaching Hospital, Faculty of Medicine, Cairo University, Cairo, Egypt \\ ${ }^{2}$ Department of Physical Therapy for Pediatrics, Faculty of Physical Therapy, Cairo University, Cairo, Egypt \\ ${ }^{3}$ Department of Basic Sciences, Faculty of Physical Therapy, Cairo University, Cairo, Egypt \\ ${ }^{4}$ Department of Physical Therapy and Health Rehabilitation, College of Applied Medical Sciences, \\ Prince Sattam Bin Abdulaziz University, Alkharj, Saudi Arabia
}

\begin{abstract}
Objectives: This study aims to compare the effect of aquatic-based exercises (AQBEs) and land-based exercises (LBEs) on muscle strength, fatigue and quality of life (QoL), and skin disease activity in children with juvenile dermatomyositis (JDM).

Patients and methods: The design of the study was an assessor-blinded, controlled $2 \times 2$ crossover trial. Fourteen children (4 boys, 10 girls; mean age $11.7 \pm 2.2$ years; range, 10 to 16 years) were evaluated. AQBEs and LBEs were applied through two treatment sequences as half of the children received AQBEs first while the second half received LBEs first. Isometric muscle strength, fatigue level and QoL (Pediatric Quality of Life Inventory Multidimensional Fatigue Scale [PedsQL-MFS]), and skin disease activity score (DAS $S_{\text {skin }}$ ) were measured at four occasions for each treatment sequence.

Results: The AQBEs had significant superiority over LBEs with improved hip flexors' strength $(p=0.007)$ and hip abductors' strength ( $p=0.001)$, while both types of treatment had the same effect in increasing strength of shoulder flexors and abductors $(p<0.05)$. AQBEs improved PedsQL-MFS, and $D_{A S}$ skin significantly more than LBEs $(p<0.001)$. For all outcome measures, there was no significant difference in the treatment sequence the children received first.

Conclusion: Supervised AQBEs are more effective in improving muscle strength, fatigue and QoL, and skin disease activity than LBEs in children with JDM. Furthermore, the treatment sequence had no significant effect on measured variables.

Keywords: Aquatic-based exercises, juvenile dermatomyositis, land-based exercises, skin disease activity.
\end{abstract}

Idiopathic inflammatory myopathies (IIMs) account for a different assemble of autoimmune diseases. ${ }^{1}$ Juvenile dermatomyositis (JDM) is the commonest IIM, responsible for nearly $85 \%$ of conditions. ${ }^{2,3} \mathrm{JDM}$ is a rare but potentially serious systemic autoimmune vasculopathy primarily affecting skin and muscles; the rate of the annual diagnosis of JDM worldwide is three in a million children, with girls to boys' ratio of approximately $3: 1.4,5$ The mean age of onset is between 7 to 18 years, although about $25 \%$ of conditions with JDM are below the age of five years. ${ }^{6}$

In JDM, the onset of muscle involvement can lead to progressive weakness in the large muscles around the shoulder and hip joints, fatigue, and myalgias that contribute to the development of

Received: April 08, 2019 Accepted: May 09, 2019 Published online: October 17, 2019

Correspondence: Ahmed Samhan, MD. Department of Physical Therapy, New Kasr El-Aini Teaching Hospital, Faculty of Medicine, Cairo University, 11562 Cairo, Egypt. Tel: 0545159701 e-mail: ahmedsamhan44@yahoo.com 
sustained physical disability impacting seriously on quality of life (QoL) in the long-term. The underlying causes of muscle weakness are not fully understood. However, it could be explained by the effect of myositis in the acute stage, metabolic alteration (i.e., decreased adenosine triphosphate concentration in muscle tissue) in the post-acute stage, ${ }^{7}$ or steroids-induced myopathy and low physical activities in the chronic stage. ${ }^{8}$ In addition, with an eruption of skin lesions, heliotrope rash, Gottron's papules and calcinosis are manifested, ${ }^{9}$ and other symptoms including dysphagia, dysphonia, abdominal pain, arthralgia, and lung problems may also be associated with JDM. ${ }^{10}$

Anecdotally, glucocorticoids and immunosuppressive drugs are effective options. ${ }^{8}$ However, use of these medications for a long time has been attributed to many adverse effects, such as osteoporosis, muscle atrophy, and cardiovascular disorders. ${ }^{11}$ The role of exercises in the therapeutic regimen for children with JDM is significant. ${ }^{12,13}$ In the past, the use of exercises in the treatment of children with JDM was prohibited, as exercises were thought to worsen muscular inflammation and accelerate the disease activity. Recently, exercises have been prescribed to enhance muscle or at the very least to maintain muscle strength and to restore function without influencing any aspect of the disease activity. ${ }^{14,15}$

Aquatic exercises are an alternative, safe and useful mode of exercises that have been widely used in the treatment of children with chronic musculoskeletal disorders. ${ }^{16}$ They help in reducing pain and muscle spasms, maintaining or increasing the range of motion, strengthening of muscle weakness, retraining of muscle paralysis, improving cutaneous blood flow, enhancing lung function, and speech as well as assisting in maintaining and improving balance, coordination, and posture. ${ }^{17}$

Aquatic exercises may enable patients to exercise more freely in water than on land. Weight release and comfort of motion permit a safe environment for movement, strengthening exercises with a decreased level of joint resistance, providing easy surroundings for patients. Despite the improvement achieved by using different treatment modalities among patients with JDM, searching for safe and efficient therapeutic interventions remains a major challenge. Therefore, in this study, we aimed to compare the effect of aquatic-based exercises (AQBEs) and land-based exercises (LBEs) on muscle strength, fatigue and QoL, and skin disease activity in children with JDM.

\section{PATIENTS AND METHODS}

This study was an assessor-blinded, controlled $2 \times 2$ crossover trial conducted at Physical Therapy Department, New Kasr Elaini Hospital, Faculty of Medicine, Cairo University, Egypt between June 2017 and February 2019. Seventeen subjects with JDM followed-up at the pediatric rheumatology clinic at $\mathrm{Abu}$ El-rish Pediatric Hospital of Cairo University and other tertiary referral hospitals across the country were invited to participate in the present study. Sixteen subjects agreed to participate, while two withdrew during the first training period due to unknown reasons. Therefore, in this setting, 14 children ( 4 boys, 10 girls; mean age $11.7 \pm 2.2$ years; range, 10 to 16 years) were assigned to one of two groups based on two treatment sequences children followed. The first group $(n=7)$ received first treatment sequence starting by LBEs followed by AQBEs with a one-month washout period in-between, and the second group $(n=7)$ received second treatment sequence which began with AQBEs followed by LBEs interspersed with a one-month washout period (Figure 1). The assessment was carried out by an independent investigator who was not aware of the sequence or the type of treatment before each evaluation session. The study protocol was approved by the Cairo University Hospitals' Ethics Committee (PTA-2017-0089). A written informed consent was obtained from authorized guardians of each patient. The study was conducted in accordance with the principles of the Declaration of Helsinki.

Subjects were included if the pediatric rheumatologist/immunologist confirmed that they fulfilled the criteria described by Bohan and Peter for diagnosis of $\mathrm{JDM}^{18}$ and the international consensus of the diagnostic criteria for JDM. Those criteria were proximal muscle weakness; characteristic skin rash; elevated skeletal muscle enzymes like aldolase, creatine kinase, transaminase, and lactate dehydrogenase; magnetic resonance imaging 


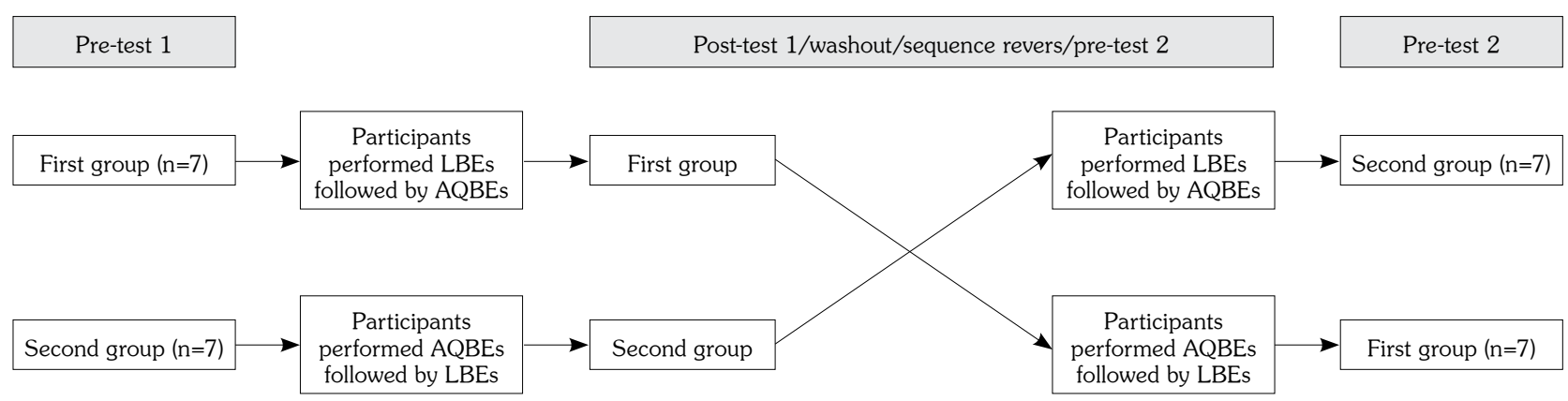

Figure 1. Flowchart illustrating crossover study design. AQBEs: Aquatic-based exercises; LBEs: Land-based exercises.

changes documenting inflammatory myositis; muscle biopsy changes of typical of myositis; electromyography changes characteristics of myopathy, and cutaneous changes such as heliotrope rash with violaceous erythema and Gottron's sign. ${ }^{19}$ Children were determined as chronic JDM (disease duration $>1$ year) 20 and identified as stable cases (i.e., received stable doses of medications for at least one month before enrollment and not in need to change the pharmacological care during the study). Children could achieve at least antigravity strength of the proximal muscle groups and were not engaged in regular exercise training at least six months before the study. Subjects were excluded if the pediatric rheumatologist recommended against participation in exercises in case of any recent relapse or presence of concurrent health-related illnesses in which the symptoms may worsen by exercises such as heart and blood vessels diseases (e.g., cardiac arrhythmias, pericarditis or myocarditis, arterial hypertension or heart failure), undernourishment, and chronic pulmonary diseases.

All children were screened for the overall disease activity using Patient Global Assessment (PGA). ${ }^{21}$ They rated their global health on a 0-10 visual analog scale. Higher scores indicated a higher level of disease activity or worse global health. The intended definition of low global assessment was $\leq 2.0$ (scale $0-10$ ).

The sample size estimation was calculated based on an expected effect size $(\mathrm{d}=0.3)$ with power of $80 \%$ and probability of 0.05 . The analysis created a sample size of 17 children; however, only 14 children were analyzed after three children were withdrawn. The analysis was performed via $G *$ Power version 3.0.10 software (Heinrich-Heine-University of Düsseldorf, Düsseldorf, Germany).

Children were assessed for isometric muscle strength, fatigue, QoL, and skin disease activity score (DAS skin) at four occasions for each treatment sequence. Two occasions, before (Pre-1) and after (Post-1), were measured in the first part of a sequence. After the washout period, another two periods, before (Pre-2) and after (Post-2), were measured in the second part of a sequence.

The isometric strength of four muscle groups including the shoulder flexors and abductors as a representation of the proximal muscles of the upper extremity and hip flexors and abductors as a representation of the proximal muscles of the lower extremity were assessed in this study.

The measurements were performed using a Lafayette hand-held dynamometer (01163-Manual Muscle Tester, Lafayette Instrument Company, Lafayette, IN, USA). The same examiner assessed all subjects, and the assessment was repeated for each child at 15-minute intervals by the same way to ensure repeatability of the measurements. ${ }^{13}$ Prior to the actual measurement, each subject was oriented to the test procedure. To measure the shoulder flexors, subjects assumed the sitting position and held the shoulder joint in $90^{\circ}$ flexion and external rotation, and the examiner placed the dynamometer on the arm at the lateral supracondylar ridge of the humerus. In measuring the shoulder abductors, subjects assumed a sitting position on a chair holding the trunk vertical while the shoulder in $90^{\circ}$ abduction, elbow in semi-flexion, and forearm in pronation, a belt was 
applied to the chest to prevent the trunk from compensatory tilting, and the dynamometer was positioned on the arm at the lateral supracondylar ridge of the humerus. To measure the hip flexors, subjects adopted sitting position with the hip and knee in $90^{\circ}$ flexion, and the feet kept off the ground to eliminate compensation by the calf muscle, the dynamometer was positioned on the anterior aspect of the distal thigh just above the superior pole of the patella. In measuring the hip abductors, subjects assumed a side-lying position with the tested limb at uppermost, the untested limb in $45^{\circ}$ hip flexion and $90^{\circ}$ knee flexion, a belt was used to stabilize the pelvis, the dynamometer was placed immediately proximal to the lateral malleolus while the hip in $0^{\circ}$ extension or abduction.

For each muscle group, strength was measured using the "make" test, as the subjects were instructed to develop muscle maximal contraction gradually. ${ }^{22}$ Subjects were required to perform two trials of three-five seconds of submaximal contraction ( 50\%) to warm up the muscle followed by three trials of five seconds of maximal effort separated by 10 -second intervals of rest. Also, an interval of 60 seconds was given between muscles groups. The mean strength (lbs) of the three trials was estimated and used in data analysis.

The Arabic version of the self-reported Pediatric Quality of Life Inventory Multidimensional Fatigue Scale (PedsQL-MFS) was used to measure fatigue in this study. ${ }^{23}$ It has been indicated as a valid and reliable measure of fatigue in children and adolescents with rheumatic diseases aged between 2-18 years. ${ }^{24}$ The scale consists of 18 items comprising three dimensions, each of which including six items. The dimensions are: general fatigue subscale, sleep/rest fatigue subscale, and cognitive fatigue subscale. Each item was scored on five-point Likert scale from 0 to 4 adopted for children (ages 8-12 years) and adolescents (ages $13-18$ years) where $0=$ never a problem, $1=$ almost never, $2=$ sometimes, $3=$ often, and $4=$ almost always a problem. Items were reversed scored and linearly transformed to a 0-100 scale as follows: $0=100,1=75,2=50,3=25,4=0$. The score by dimension was calculated as the sum of the items divided by the number of items answered in each dimension. The total score was calculated as the sum of all the items divided by the number of items answered on all dimensions. Lower scores indicated high intense fatigue and higher scores indicated low intense fatigue.

The disease activity score (DAS) was employed for the assessment of JDM skin activity. The DAS exhibited evidence of good validity and reliability in the evaluation of cutaneous and muscular changes in children with JDM. ${ }^{25}$ It is a 19 -item instrument, resulting in a sum score ranging from 0 to 20 through $\mathrm{DAS}_{\text {skin }}$ subsection (range 0-9) and $\mathrm{DAS}_{\text {muscles }}$ subsection (range 0-11). The items are scored based on whether the disease indicator is present or absent and severity level. Higher score indicates greater disease activity. The assessment was conducted by a dermatologist who had eight years of experience in the field and spent two years in allergy/immunology fellowship.

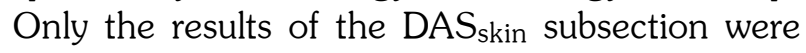
used for the statistical analysis.

Aquatic exercises were conducted in a therapeutic HydroWorx ${ }^{\circledR}$ pool (HydroWorks, PA, USA) of $8 \times 12$ feet with accessories like inclines and removable parallel bars. Water temperature was kept at $32^{\circ} \mathrm{C}$ but could be ranged from $31-34^{\circ} \mathrm{C}$. The height of the water in the pool changed according to the requirements of each child. For children who needed further support for upright, erect posture, the height of water was mainly at the level of the axilla.

The training lasted approximately for 45 minutes and was repeated twice/week for four successive weeks. The program consisted of three components as follows:

Warming up: It was a 10 -minute process in the form of light aerobic warm-up (i.e., a treadmill walking or a cycle ergometer training at an intensity adjusted to $50 \%$ of the target heart rate, dynamic stretching exercises, active range of motion exercises, and free walking at the shallow side of the pool.

Exercises loading: It was the major portion of the program that was applied for 30 minutes. The training was initiated by movement activities while standing at the shallow end, followed by reciprocal arm and leg movement, arm and leg circle, running in place, jumping up in place, running from one side of the pool to the other side. Then, the children performed strengthening activities for the upper and lower limbs using water resistance and aquatic noodles including straight 
leg and arm kicks forward, backward and to the sides; straight knee kicks; scissor kicks; arm and leg arm press; rowing and reverse rowing; knee bend; front/backswing; side swing, hip circle; and cycling. During strength training, children were instructed to move their arm/leg with the maximum effort to achieve the highest speed. ${ }^{16}$ Targeted muscles were the proximal muscles of the shoulder and hip joints, specifically the flexor and abductor muscles.

Cooling down: It was a five-minute period during which free low-intense water exercises were performed at a slow pace while stretching exercises were also administered.

In this program, children exercised individually twice/week under the supervision of a physical therapist for four consecutive weeks. The training commenced with 10-minute warming up including a treadmill walking at an intensity started from $25 \%$ and gradually increased to $50 \%$ of the target heart rate and stretching exercises. After that, children performed 30-minutes of aerobic exercises (15 minutes) and strength training (15 minutes). The treadmill speed in aerobic training was set at a speed corresponding to $75 \%$ of maximum oxygen uptake that was determined from a priori maximal exercise test. In strength training, the exercise load gradually progressed from low-intensity isometric exercises and active free exercises to mild or moderate resistive exercises depending on each child response. For the resistive training, each child performed two sets of 10-15 repetition maximum (RM) increased to three sets of 5-10 RM in the subsequent sessions for five muscle groups including the hip flexor and abductors, shoulder flexor and abductors, and quadriceps using free weights. The training also included instrumented-based exercises which were the leg press, leg extension, seated rowing exercises, bench press, and latissimus dorsi pull down exercises. The sets of resistance were separated by 30 -second rest. Finally, children performed a five-minute cooling down in the form of stretching exercises.

The dosage of the drugs administered depended on the severity of the disease. A similar protocol of medication was followed: (i) immunosuppressant (methotrexate, $1 \mathrm{mg} / \mathrm{kg}$ once per week, with a maximum dose of $40 \mathrm{mg}$ ); (ii) corticosteroids (prednisone, $2 \mathrm{mg} / \mathrm{kg} / \mathrm{day}$, once a day for four weeks, with maximum dose of $60 \mathrm{mg}$, which was reduced by $20 \%$ in the next weeks); (iii) intravenous immune globulin (2 gm/kg every two weeks for three months, with a maximum dose of $70 \mathrm{mg}$, thereafter it was administered on a monthly basis.

\section{Statistical analysis}

The statistical tests were conducted using IBM $^{\circledR}$ SPSS version 24.0 software for Windows (IBM Corp., Armonk, NY, USA). Data were assessed for normal distribution using the Shapiro-Wilk test. All outcome measures (Pre-1, Post-1, Pre-2, and Post-2 variables) were taken as dependent variables. Therefore, these variables were analyzed using a two-way withinsubjects repeated measures analysis of variance (ANOVA) test. Data were considered significant at $\mathrm{p}<0.01$.

\section{RESULTS}

Fourteen children of both sexes were included in the study (71\% girls and $29 \%$ boys). Their

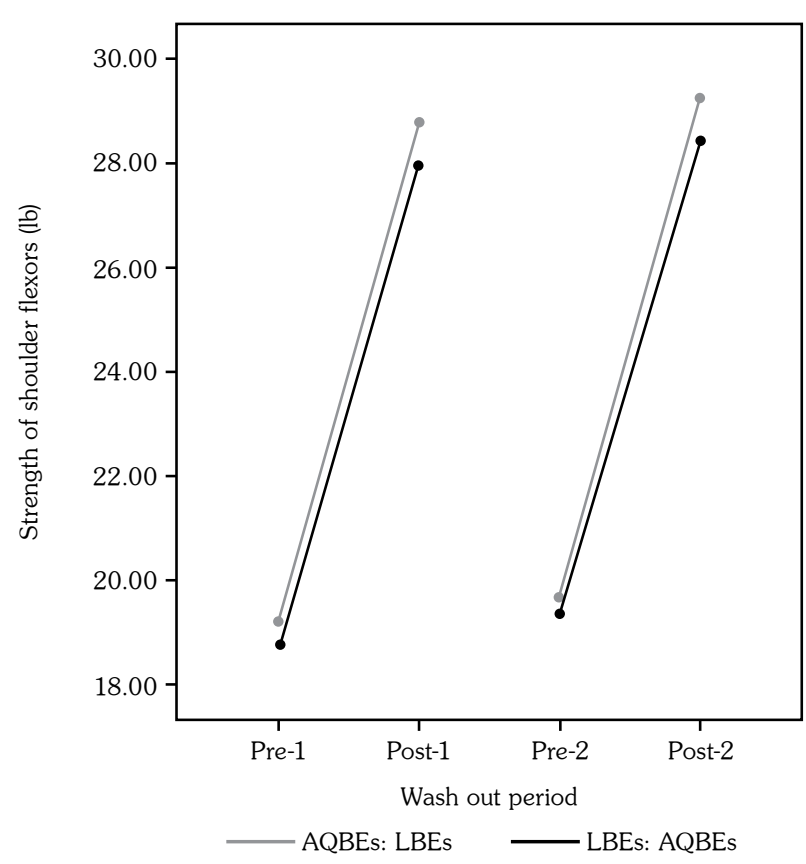

Figure 2. Strength changes of shoulder flexors due to application of aquatic-and land-based exercises.

Pre: Preoperative; Post: Postoperative; AQBEs: Aquatic-based exercises; LBEs: Land-based exercises; Pre-1: Before first part of a sequence; Post-1: After first part of a sequence; Pre-2: Before second part of a sequence; Post-2: After second part of a sequence. 
mean disease duration was $4.6 \pm 1.1$ years. The mean level of disease activity measured by PGA was $5.8 \pm 1.2$. After the end of both treatments, all 14 patients correctly finished the treatment sessions. Results were based on two-way withinsubjects repeated measures ANOVA.

There was a significant improvement of shoulder flexors' strength ( $F 3,4=130, p<0.001$; Wilks' $\Lambda=0.025$, partial $\left.\eta^{2}=0.975\right)$ in both AQBEs and LBEs groups. However, there was no statistical significance ( $p>0.05$ ) that suggested the superiority of one type of exercise over the other (Figure 2). The strength of shoulder abductors behaved similarly in both types of treatment (F 3,4=52.6, $\mathrm{p}<0.001$; Wilks' $\Lambda=0.06$, partial $\left.\eta^{2}=0.940\right)$ with no superiority of one treatment ( $p>0.05)$ (Figure 3).

The strength of hip flexors improved significantly in both AQBEs and LBEs groups (F 3,4=138.2, $p<0.001$; Wilks' $\Lambda=0.01$, partial $\left.\eta^{2}=0.990\right)$ but with a preference for the AQBEs $(32 \pm 2.1 \mathrm{lb})$ over LBEs $(25.1 \pm 4.3 \mathrm{lb})(p=0.007)$ (Figure 4). As for the hip abductors, there was a

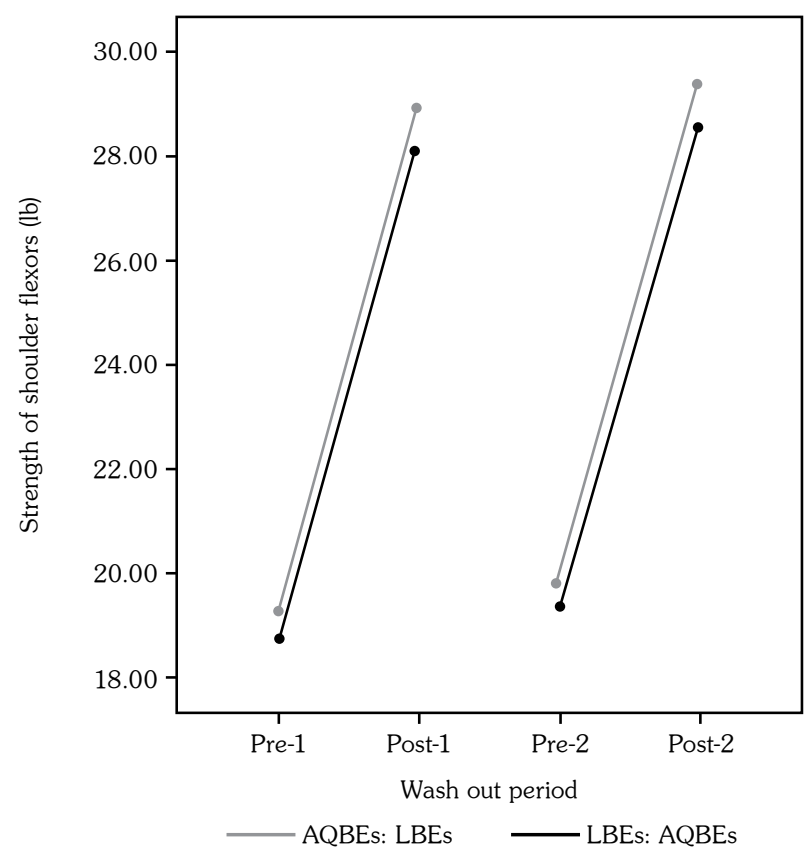

Figure 3. Effect of aquatic- and land-based exercises on shoulder abductors.

Pre: Preoperative; Post: Postoperative; AQBEs: Aquatic-based exercises; LBEs: Land-based exercises; Pre-1: Before first part of a sequence; Post-1: After first part of a sequence; Pre-2: Before second part of a sequence; Post-2: After second part of a sequence. significant increase in strength after the application of AQBEs and LBEs ( $F$ 3,4=51.4, $\mathrm{p}=0.001$; Wilks' $\Lambda=0.025$, partial $\eta^{2}=0.975$ ); however, the strength of hip abductors $(31.4 \pm 2.2 \mathrm{lb})$ increased significantly by AQBEs compared with LBEs $(23.9 \pm 4.8 \mathrm{lb})(p=0.012)$ (Figure 5). The sequence of treatment which the children followed did not affect significantly the strength of hip flexors (F 1,6 $=0.012, p=0.915$; Wilks' $\Lambda=0.998$, partial $\left.\eta^{2}=0.002\right)$ and hip abductors (F 1,6 $=0.311$, $p=0.597$; Wilks' $\Lambda=0.951$, partial $\eta^{2}=0.049$ ).

There was a significant decrease in the general fatigue measured by PedsQL-MFS in both AQBEs and LBEs groups (F 3,4=517.6, p<0.001; Wilks' $\Lambda=0.003$, partial $\left.\eta^{2}=0.997\right)$. Nevertheless, AQBEs improved the PedsQL-MFS more than LBEs $(44.15 \pm 2.65,61.9 \pm 3.9$, respectively) $(p<0.001)$. Figure 6 demonstrates the PedsQL-MFS changes between both treatments. General fatigue score was not significantly affected according to the sequence of treatment given first $(F$ 1,6 $=2.1$, $\mathrm{p}=0.2$; Wilks' $\Lambda=0.743$, partial $\eta^{2}=0.26$ ).

Although both types of treatments enhanced the overall disease activity significantly

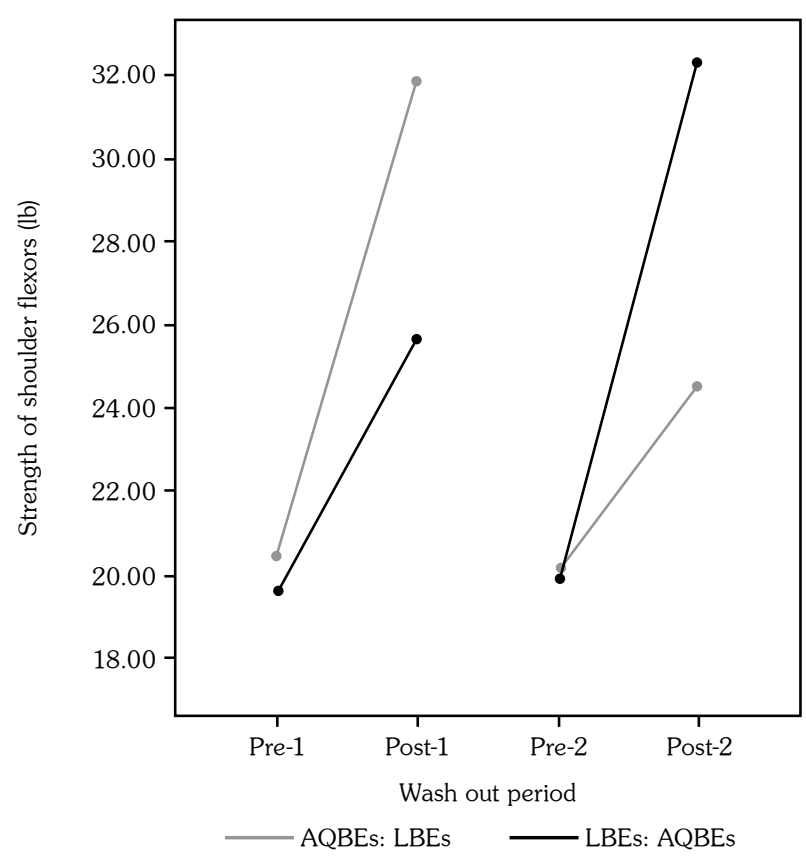

Figure 4. Effect of aquatic- and land-based exercises on the hip flexors.

Pre: Preoperative; Post: Postoperative; AQBEs: Aquatic-based exercises; LBEs: Land-based exercises; Pre-1: Before first part of a sequence; Post-1: After first part of a sequence; Pre-2: Before second part of a sequence; Post-2: After second part of a sequence. 


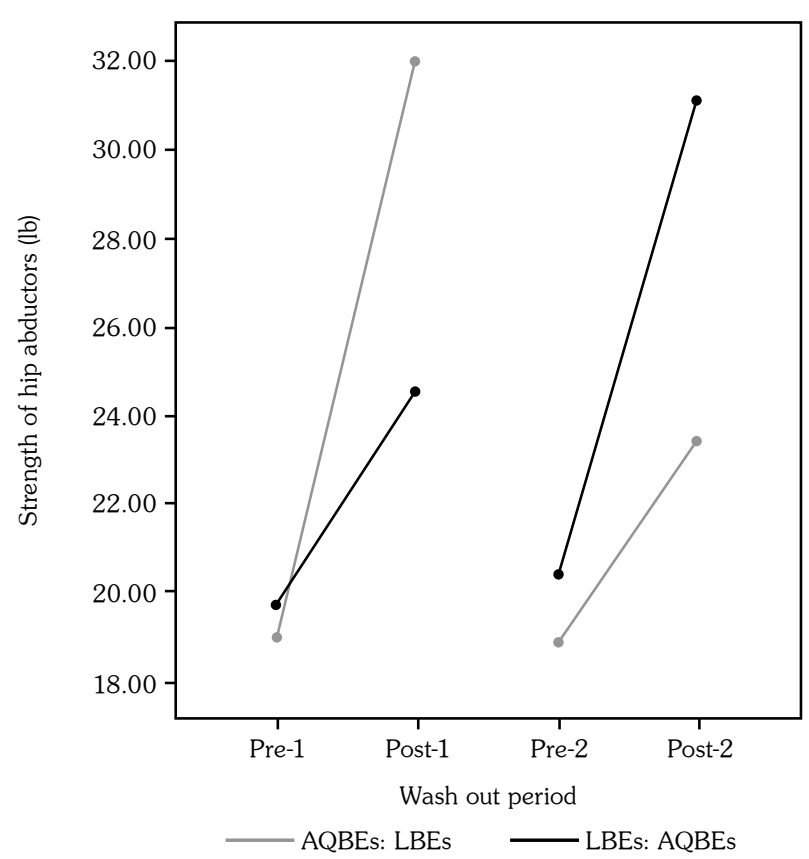

Figure 5. Strength changes of hip abductors due to aquatic- and land-based exercises.

Pre: Preoperative; Post: Postoperative; AQBEs: Aquatic-based exercises; LBEs: Land-based exercises; Pre-1: Before first part of a sequence; Post-1: After first part of a sequence; Pre-2: Before second part of a sequence; Post-2: After second part of a sequence.

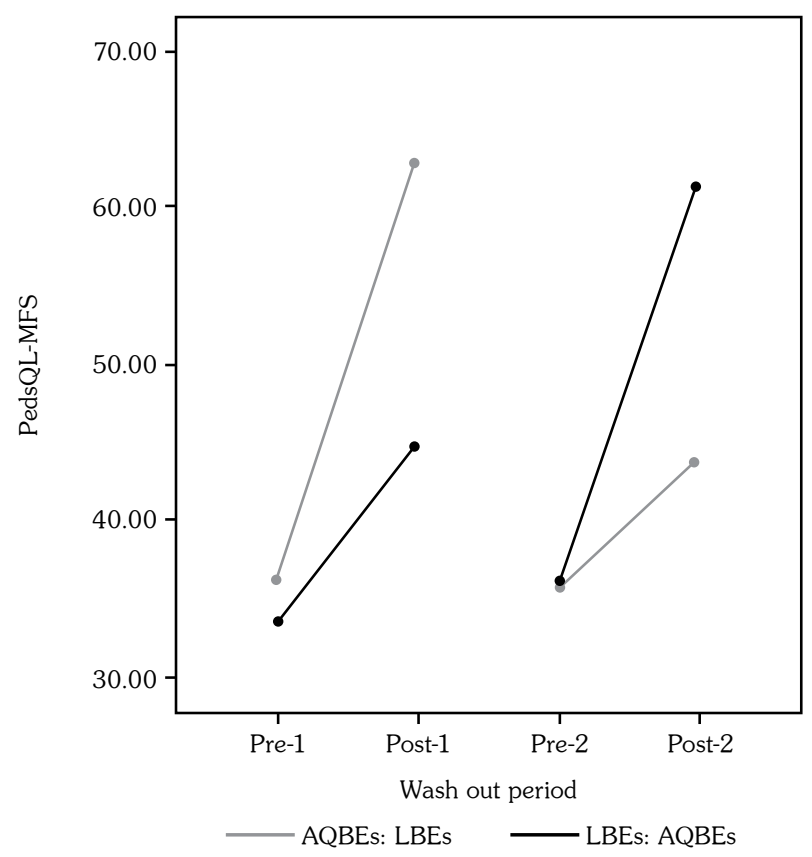

Figure 6. Effect of aquatic- and land-based exercises on general fatigue.

Pre: Preoperative; Post: Postoperative; AQBEs: Aquatic-based exercises; LBEs: Land-based exercises; PedsQL-MFS: Pediatric Quality of Life Inventory Multidimensional Fatigue Scale; Pre-1: Before first part of a sequence; Post-1: After first part of a sequence; Pre-2: Before second part of a sequence; Post-2: After second part of a sequence.

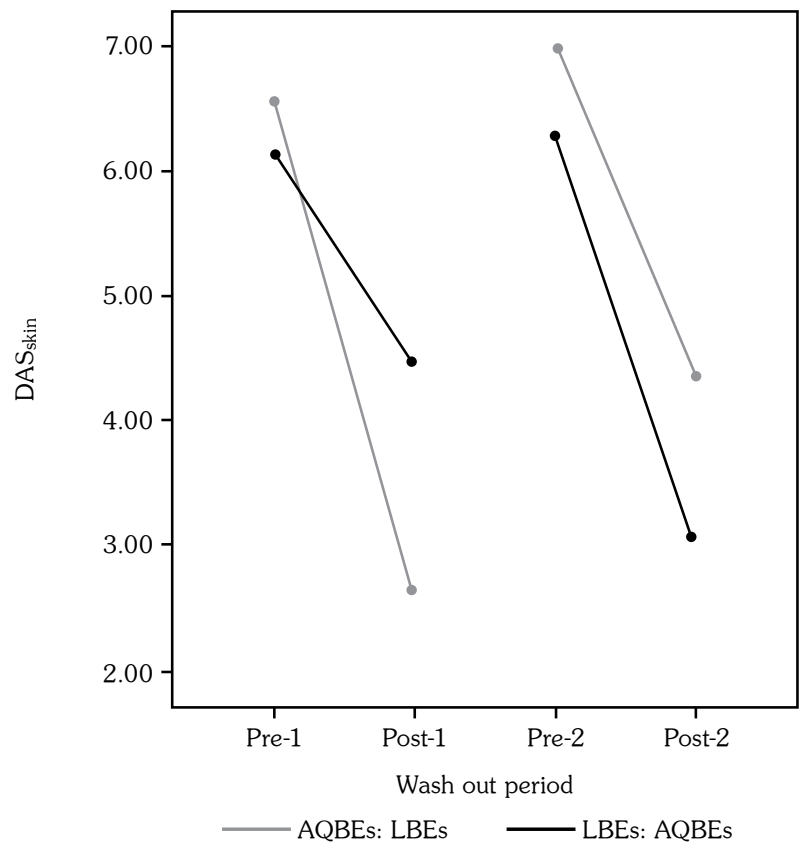

Figure 7. Effect of aquatic- and land-based exercises on skin disease activity score.

Pre: Preoperative; Post: Postoperative; AQBEs: Aquatic-based exercises; LBEs: Land-based exercises; DAS ${ }_{\text {skin: }}$ Skin disease activity score; Pre-1: Before first part of a sequence; Post-1: After first part of a sequence; Pre-2: Before second part of a sequence; Post-2: After second part of a sequence.

(F 3,4=236.9, p<0.001; Wilks' $\Lambda=0.006$, partial $\left.\eta^{2}=0.994\right)$, AQBEs program showed a marked advantage over LBEs in terms of DAS skin $(2.8 \pm 1.3, \quad 4.35 \pm 1.8$, respectively) $(p=0.011)$ (Figure 7). There was no significant influence of the treatment sequence on $\mathrm{DAS}_{\text {skin }}(\mathrm{F} 1,6=0.06$, $\mathrm{p}=0.822$; Wilks' $\Lambda=0.991$, partial $\eta^{2}=0.01$ ).

\section{DISCUSSION}

Juvenile dermatomyositis is a consequential sickness that induces proximal muscle weakness, fatigue, lack of physical activity, and skin diseases. Since the establishment of new treatments, the concentration of outcome measures has transferred from mortality towards morbidity, functional activity, fatigability, QoL, and skin disease activity. ${ }^{26,27}$ This study had two main objectives. First was to compare AQBEs versus LBEs in improving proximal muscles strength of upper and lower extremities, general fatigue and QoL, and skin disease activity (DAS skin score) in children with JDM. Second was to evaluate the influence of the treatment 
sequence in the same outcome measures in children with JDM. The findings suggested that AQBEs have superiority over LBEs in increasing hip flexors and abductors muscles' strength, decreasing general fatigue using PedsQL-MFS, and improving DAS skin scores, while there were no differences in the treatment sequence starting with aquatic or land exercises. On the other hand, there was a similar improvement of shoulder flexors and abductors muscles' strength in both types of exercises.

To our knowledge, this was the first trial to compare the impacts of AQBEs versus LBEs and to evaluate the ideal treatment sequence in children with JDM. The findings of this study determined that AQBEs can counteract a wide extent of manifestations in children with JDM, comprising muscle weakness, fatigue, and skin conditions. Consequentially, a significant improvement in QoL was also detected with no aggravation of disease activity. Altogether, these findings provide evidence recommending that $\mathrm{AQBEs}$ are both safe and effective for children with JDM.

In accordance with Takken et al., ${ }^{27}$ there are various pathophysiologic interpretations for the apparent deterioration in exercise tolerance in children with JDM: the raised level of intramuscular cytokines, the generalized inflammatory mechanisms, the inflammation of muscular blood vessels, physical inactivity, the damaged muscle oxidative capacity, and the influence of corticosteroids therapy on acquiring body weight and protein destruction. ${ }^{27,28}$ In the past, physical exercises such as muscle strengthening exercises have been contraindicated in patients with JDM. It was considered that physical exercise program may lead to impairment of muscle fiber and increased inflammatory process in JDM patients. ${ }^{29}$ Nevertheless, new evidence proposed that a physical exercise program for patients with inactive JDM does not increase the inflammatory process of muscle fibers. ${ }^{30}$ The findings of this study further support this idea, indicating that AQBEs were tolerable in JDM children.

The AQBEs were capable of remarkably enhancing the lower and upper extremity muscles strength (hips and shoulders' flexors and abductors) and general fatigue (PedsQL-MFS). Engagingly, the advancements in the clinical evaluations of muscle strength and general fatigue were paralleled by the advancements in $\mathrm{DAS}_{\text {skin }}$ in children with JDM because of the positive influence of water in increasing cutaneous blood flow and improving skin conditions.

In a study by Faigenbaum et al. ${ }^{31}$ to evaluate the impacts of resistance exercises in healthy pre-maturational children, there was a neural improvement (inter- and intramuscular coordination) instead of structural variables (crosswise muscle area and fiber types). Particularly, the advantages of physical exercises in the neuromuscular system may be of objective importance in the treatment of patients with $\mathrm{JDM}$, as both muscle wasting and weakness are of great interests for children with JDM. ${ }^{13}$

The findings of this study suggest that AQBEs are effective alternative exercises compared to LBEs for children JDM. Aquatic exercises in children are characterized by further cheerful and non-weight-bearing exercises, requiring less exertion and providing more painless exercises, with low possibility of injury compared with traditional exercises. ${ }^{32}$ These characteristics allow children to consider aquatic exercises as playing rather than exercising. Satisfaction is important because it motivates a child to be involved in the exercise program, increases the child's energy degree, and stimulates him/her to keep good condition. ${ }^{33}$ Aquatic exercises use the physical characteristics of water to perform specific movements for weakened muscles which may be impossible to apply on land. They permit children to practice antigravity movement schemes and patterns using the resistive force of buoyancy in water. Moreover, the viscous drag allows performing a wide spectrum of strengthening exercises that can be adopted easily by children. ${ }^{16,34}$ The improved DAS $_{\text {skin }}$ may be attributed to the curing characteristics of water with its temperature and physical effects. The water over the skin stimulates the peripheral nervous system in enhancing the immune system, increasing circulation, affecting the output of stress hormones, increasing the pain threshold as well as the skin blood flow. ${ }^{35}$

The findings of this study are limited by the study design as crossover studies need a washout period between both treatment phases. Moreover, because JDM is a rare disease, a small sample 
size was included; however, our findings were consistent and highly significant. We attempted to overcome the small sample issue by randomizing the participants into two treatment sequences. Finally, the efficacy of aquatic exercises was evaluated only in the short-term; thus, future studies are needed to ascertain if the impacts of this therapy are continued in the long-term such as six months or further.

In conclusion, supervised aquatic exercises are safe and more effective in improving muscle strength, fatigue and QoL, and skin disease activity than LBEs in children with JDM. Furthermore, clinically, the treatment sequence resulted in no differences, as beginning with aquatic or landing exercises provided the same effects. We hope that these findings contribute to the growing body of knowledge indicating the positive therapeutic role of aquatic exercises in the treatment procedures for children with JDM.

\section{Acknowledgments}

The authors thank all children and their parents for their cooperation in this study as well as the staff of Department of Physical Therapy New Kasr Elaini Teaching Hospital for their assistance and motivation.

\section{Declaration of conflicting interests}

The authors declared no conflicts of interest with respect to the authorship and/or publication of this article.

\section{Funding}

The authors received no financial support for the research and/or authorship of this article.

\section{REFERENCES}

1. Wedderburn LR, Rider LG. Juvenile dermatomyositis: new developments in pathogenesis, assessment and treatment. Best Pract Res Clin Rheumatol 2009;23:665-78.

2. McCann LJ, Juggins AD, Maillard SM, Wedderburn LR, Davidson JE, Murray KJ, et al. The Juvenile Dermatomyositis National Registry and Repository (UK and Ireland)--clinical characteristics of children recruited within the first 5 yr. Rheumatology (Oxford) 2006;45:1255-60.

3. Ravelli A, Trail L, Ferrari C, Ruperto N, Pistorio A, Pilkington $\mathrm{C}$, et al. Long-term outcome and prognostic factors of juvenile dermatomyositis: a multinational, multicenter study of 490 patients. Arthritis Care Res (Hoboken) 2010;62:63-72.
4. Mendez EP, Lipton R, Ramsey-Goldman R, Roettcher $\mathrm{P}$, Bowyer S, Dyer A, et al. US incidence of juvenile dermatomyositis, 1995-1998: results from the National Institute of Arthritis and Musculoskeletal and Skin Diseases Registry. Arthritis Rheum 2003;49:300-5.

5. Symmons DP, Sills JA, Davis SM. The incidence of juvenile dermatomyositis: results from a nation-wide study. Br J Rheumatol 1995;34:732-6.

6. Martin N, Krol P, Smith S, Murray K, Pilkington CA, Davidson $\mathrm{JE}$, et al. A national registry for juvenile dermatomyositis and other paediatric idiopathic inflammatory myopathies: 10 years' experience; the Juvenile Dermatomyositis National (UK and Ireland) Cohort Biomarker Study and Repository for Idiopathic Inflammatory Myopathies. Rheumatology (Oxford) 2011;50:137-45.

7. Cea G, Bendahan D, Manners D, Hilton-Jones D, Lodi $\mathrm{R}$, Styles $\mathrm{P}$, et al. Reduced oxidative phosphorylation and proton efflux suggest reduced capillary blood supply in skeletal muscle of patients with dermatomyositis and polymyositis: a quantitative 31P-magnetic resonance spectroscopy and MRI study. Brain 2002;125:1635-45.

8. Zong M, Lundberg IE. Pathogenesis, classification and treatment of inflammatory myopathies. Nat Rev Rheumatol 2011;7:297-306.

9. Hicks JE, Drinkard B, Summers RM, Rider LG. Decreased aerobic capacity in children with juvenile dermatomyositis. Arthritis Rheum 2002;47:118-23.

10. Rider LG, Wu L, Mamyrova G, Targoff IN, Miller FW. Environmental factors preceding illness onset differ in phenotypes of the juvenile idiopathic inflammatory myopathies. Rheumatology (Oxford) 2010;49:2381-90.

11. Poetker DM, Reh DD. A comprehensive review of the adverse effects of systemic corticosteroids. Otolaryngol Clin North Am 2010;43:753-68.

12. Harris-Love MO. Physical activity and disablement in the idiopathic inflammatory myopathies. Curr Opin Rheumatol 2003;15:679-90.

13. Omori $\mathrm{CH}$, Silva $\mathrm{CA}$, Sallum AM, Rodrigues Pereira $\mathrm{RM}$, Lúciade Sá Pinto A, Roschel H, et al. Exercise training in juvenile dermatomyositis. Arthritis Care Res (Hoboken) 2012;64:1186-94.

14. Alexanderson H, Dastmalchi M, EsbjörnssonLiljedahl M, Opava $\mathrm{CH}$, Lundberg IE. Benefits of intensive resistance training in patients with chronic polymyositis or dermatomyositis. Arthritis Rheum 2007;57:768-77.

15. Arnardottir S, Alexanderson H, Lundberg IE, Borg K. Sporadic inclusion body myositis: pilot study on the effects of a home exercise program on muscle function, histopathology and inflammatory reaction. J Rehabil Med 2003;35:31-5.

16. Elnaggar RK, Elshafey MA. Effects of Combined Resistive Underwater Exercises and Interferential Current Therapy in Patients with Juvenile Idiopathic Arthritis: A Randomized Controlled Trial. Am J Phys Med Rehabil 2016;95:96-102. 
17. Cole AJ, Becker BE. Comprehensive aquatic therapy. Philadelphia: Butterworth-Heinemann; 2004.

18. Bohan A, Peter JB. Polymyositis and dermatomyositis (first of two parts). N Engl J Med 1975;292:344-7.

19. Brown VE, Pilkington CA, Feldman BM, Davidson JE. An international consensus survey of the diagnostic criteria for juvenile dermatomyositis (JDM). Rheumatology (Oxford) 2006;45:990-3.

20. Ruperto N, Pistorio A, Ravelli A, Rider LG, Pilkington $\mathrm{C}$, Oliveira $\mathrm{S}$, et al. The Paediatric Rheumatology International Trials Organisation provisional criteria for the evaluation of response to therapy in juvenile dermatomyositis. Arthritis Care Res (Hoboken) 2010;62:1533-41.

21. Nikiphorou E, Radner $H$, Chatzidionysiou $K$, Desthieux C, Zabalan C, van Eijk-Hustings Y, et al. Patient global assessment in measuring disease activity in rheumatoid arthritis: a review of the literature. Arthritis Res Ther 2016;18:251.

22. Bohannon RW. Make tests and break tests of elbow flexor muscle strength. Phys Ther 1988;68:193-4.

23. Al-Gamal E, Long T. The psychometric properties of an Arabic version of the PedsQL multidimensional fatigue scale tested for children with cancer. Compr Child Adolesc Nurs 2017;40:188-99.

24. Varni JW, Burwinkle TM, Szer IS. The PedsQL Multidimensional Fatigue Scale in pediatric rheumatology: reliability and validity. J Rheumatol 2004;31:2494-500.

25. Bode RK, Klein-Gitelman MS, Miller ML, Lechman TS, Pachman LM. Disease activity score for children with juvenile dermatomyositis: reliability and validity evidence. Arthritis Rheum 2003;49:7-15.

26. Sallum AM, Kiss MH, Sachetti S, Resende MB, Moutinho KC, Carvalho Mde S, et al. Juvenile dermatomyositis: clinical, laboratorial, histological, therapeutical and evolutive parameters of 35 patients. Arq Neuropsiquiatr 2002;60:889-99.
27. Takken $\mathrm{T}$, van der Net J, Engelbert RH, Pater S, Helders PJ. Responsiveness of exercise parameters in children with inflammatory myositis. Arthritis Rheum 2008;59:59-64.

28. Takken T, van der Net J, Helders PJ. The reliability of an aerobic and an anaerobic exercise tolerance test in patients with juvenile onset dermatomyositis. $\mathrm{J}$ Rheumatol 2005;32:734-9.

29. de Salles Painelli V, Gualano B, Artioli GG, de Sá Pinto AL, Bonfá E, Lancha Junior $\mathrm{AH}$, et al. The possible role of physical exercise on the treatment of idiopathic inflammatory myopathies. Autoimmun Rev 2009;8:355-9.

30. Maillard SM, Jones R, Owens CM, Pilkington C, Woo PM, Wedderburn LR, et al. Quantitative assessments of the effects of a single exercise session on muscles in juvenile dermatomyositis. Arthritis Rheum 2005;53:558-64.

31. Faigenbaum AD, Kraemer WJ, Blimkie CJ, Jeffreys I, Micheli LJ, Nitka M, et al. Youth resistance training: updated position statement paper from the national strength and conditioning association. J Strength Cond Res 2009;23:S60-79.

32. Gorter JW, Currie SJ. Aquatic exercise programs for children and adolescents with cerebral palsy: what do we know and where do we go? Int $\mathrm{J}$ Pediatr 2011;2011:712165.

33. Lai CJ, Liu WY, Yang TF, Chen CL, Wu CY, Chan RC. Pediatric aquatic therapy on motor function and enjoyment in children diagnosed with cerebral palsy of various motor severities. J Child Neurol 2015;30:200-8.

34. Salem Y, Gropack SJ. Aquatic therapy for a child with type III spinal muscular atrophy: a case report. Phys Occup Ther Pediatr. 2010;30:313-24.

35. Davison PG, Loiselle FB, Nickerson D. Survey on current hydrotherapy use among North American burn centers. J Burn Care Res 2010;31:393-9. 